\title{
Dimensional Reduction and Crossover to Mean-Field Behavior for Branched Polymers
}

\author{
John Z. Imbrie \\ Department of Mathematics \\ University of Virginia \\ Charlottesville, VA 22904-4137
}

\begin{abstract}
This article will review recent results on dimensional reduction for branched polymers, and discuss implications for critical phenomena. Parisi and Sourlas argued in PS81 that branched polymers fall into the universality class of the Yang-Lee edge in two fewer dimensions. Brydges and I have proven in BI01 that the generating function for self-avoiding branched polymers in $D+2$ continuum dimensions is proportional to the pressure of the hard-core continuum gas at negative activity in $D$ dimensions (which is in the Yang-Lee or $i \varphi^{3}$ class). I will describe how this equivalence arises from an underlying supersymmetry of the branched polymer model.

I will also use dimensional reduction to analyze the crossover of twodimensional branched polymers to their mean-field limit, and to show that the scaling is given by an Airy function (the same as in Car01).
\end{abstract}

Let us begin with a definition of branched polymers, first on the lattice, then in continuous space. On the lattice, branched polymers should look like the following picture. 
Define $c_{N}$ to be the number of $N$-vertex branched polymers mod translations. Let $T$ be a tree graph on $\{1, \ldots, N\}$ and $y_{1}, \ldots, y_{N}$ be an embedding of the graph in the lattice. In other words, $y_{i}$ denotes the position of the $i^{\text {th }}$ vertex of the tree graph. If $i$ and $j$ are connected by a line in the tree graph, then $y_{i}$ and $y_{j}$ must be adjacent in the lattice. In addition, no two vertices may be mapped to the same point; this would lead to a loop. The generating function $Z_{\mathrm{BP}}(z)=\sum_{N} z^{N} c_{N}$ can be written as

$$
Z_{\mathrm{BP}}(z)=\sum_{N=1}^{\infty} \frac{z^{N}}{N !} \sum_{T} \sum_{y_{2}, \ldots, y_{N}} \prod_{i j \in T}\left[2 U_{i j}^{\prime}\right] \prod_{i j \notin T} U_{i j},
$$

where

$$
2 U_{i j}^{\prime}=\delta_{\left|y_{i}-y_{j}\right|, 1}
$$

and

$$
U_{i j}=1-\delta_{y_{i}, y_{j}}
$$

enforce the adjacency and loop-free conditions, respectively. For example, $c_{3}=$ $\frac{1}{3 !} \cdot 3 \cdot 4 \cdot 3=6$ in $\mathbb{Z}^{2}$ as there are 3 tree graphs on $\{1,2,3\}$, and for each of them there are 4 choices for $y_{2}$ and 3 for $y_{3}$. See BI01 Frö86 for details.

\section{Background}

The concept of dimensional reduction was introduced by Parisi and Sourlas in 1979 in order to understand the behavior of the Ising model in a random magnetic field PS79. Although the proof of long-range order in three dimensions \mb84 showed that dimensional reduction did not work in that problem, dimensional reduction appeared to work for branched polymers PS81. Our proof answers any lingering questions about dimensional reduction for branched polymers and gives a clear picture of how it works. The question of what goes wrong for random fields continues to be debated in the literature BD98 PS02 Fel02.

Dimensional reduction for branched polymers was employed recently by Cardy to determine the crossover scaling function for area-weighted self-avoiding loops in two dimensions Car01. Below, I will use our dimensional reduction identity to derive a similar result for the crossover from branched polymers to their noninteracting or mean-field limit.

\section{Branched Polymers in $\mathbb{R}^{D+2}$}

Lattice models of branched polymers have been written down that are supersymmetric after dropping irrelevant terms Sha83 Sha85. Here we work in continuous space so that the supersymmetry responsible for dimensional reduction can be preserved. Let us adjust the definitions given above for lattice branched polymers to the continuous case. In $\mathbb{R}^{D+2}$, a branched polymer consists of

1. A tree graph $T$ on $1, \ldots, N$ and 
2. An embedding $\mathbf{y}=\left\{y_{i}\right\}_{i=1, \ldots, N}$ into $\mathbb{R}^{D+2}$ such that

- if $i j \in T$ then $\left|y_{i j}\right|=1$ and

- if $i j \notin T$ then $\left|y_{i j}\right| \geq 1$.

The generating function for branched polymers (mod translations) is

$$
Z_{\mathrm{BP}}(z)=\sum_{N=1}^{\infty} \frac{z^{N}}{N !} \sum_{T} \int d y_{2} \cdots d y_{N} \prod_{i j \in T}\left[2 U_{i j}^{\prime}\right] \prod_{i j \notin T} U_{i j},
$$

where

$$
U_{i j}=U\left(\left|y_{i}-y_{j}\right|^{2}\right)
$$

and

$$
2 U_{i j}^{\prime}=2 U^{\prime}\left(\left|y_{i}-y_{j}\right|^{2}\right)=\delta\left(\left|y_{i}-y_{j}\right|-1\right) .
$$

As before, $U_{i j}^{\prime}$ enforces adjacency, and $U_{i j}$ is a self-avoidance condition. One can think of these branched polymers as collections of hard spheres with kissing conditions determined by the tree graph $T$.

\section{The Hard-Core Gas in $\mathbb{R}^{D}$}

We shall relate branched polymers in $\mathbb{R}^{D+2}$ to the hard-core gas in $\mathbb{R}^{D}$. The hardcore gas has hard spheres centered at $x_{i} \in \mathbb{R}^{D}$ for $i=1, \ldots, N$. Let

$$
x_{i j}=x_{i}-x_{j}, t_{i j}=\left|x_{i j}\right|^{2},
$$

and let

$$
U\left(t_{i j}\right)=\theta\left(t_{i j}-1\right)
$$

enforce the hard-core constraint. Then

$$
Z_{\mathrm{HC}}(z)=\sum_{N=0}^{\infty} \frac{z^{N}}{N !} \int_{\Lambda^{N}} d x_{1} \cdots d x_{N} \prod_{1 \leq i<j \leq N} U\left(\left|x_{i}-x_{j}\right|^{2}\right) .
$$

\section{Main Results}

Theorem 1. For all $z$ such that the right-hand side converges absolutely,

$$
\lim _{\Lambda \nearrow \mathbb{R}^{D}} \frac{1}{|\Lambda|} \log Z_{\mathrm{HC}}(z)=-2 \pi Z_{\mathrm{BP}}\left(-\frac{z}{2 \pi}\right) .
$$




\section{Examples}

Exact computation of the left-hand side is possible in $D=0,1$. For $D=0$, $Z_{\mathrm{HC}}(z)=1+z$ and so in dimension $D+2=2$,

$$
Z_{\mathrm{BP}}(z)=-\frac{1}{2 \pi} \log (1-2 \pi z) .
$$

The pressure of the one-dimensional gas of hard rods is also computable, see for example BI03. Hence in dimension $D+2=3$,

$$
Z_{\mathrm{BP}}(z)=\frac{1}{2 \pi} T(2 \pi z)=\sum_{N=1}^{\infty} \frac{N^{N-1}}{2 \pi N !}(2 \pi z)^{N} .
$$

Here $T(z)=-$ LambertW $(-z)$ is the tree function CGHJK. These equations allow us to write a simple expression for $c_{N}$ in two and three dimensions.

\section{Green's Functions}

We also have a dimensional reduction formula for correlations. First, some definitions. Let

$$
\rho(\tilde{x})=\sum_{i=1}^{N} \delta\left(\tilde{x}-x_{i}\right), \quad \rho(\tilde{y})=\sum_{i=1}^{N} \delta\left(\tilde{y}-y_{i}\right),
$$

where $\tilde{x}, x_{i} \in \mathbb{R}^{D}$ and $\tilde{y}, y_{i} \in \mathbb{R}^{D+2}$. Then the density-density correlation functions of the two systems can be written as

$$
\begin{aligned}
G_{\mathrm{BP}}\left(\tilde{y}_{1}, \tilde{y}_{2} ; z\right) & =\sum_{N=1}^{\infty} \frac{z^{N}}{N !} \sum_{T} \int d y_{1} \cdots d y_{N} \rho\left(\tilde{y}_{1}\right) \rho\left(\tilde{y}_{2}\right) \prod_{i j \in T}\left[2 U_{i j}^{\prime}\right] \prod_{i j \notin T} U_{i j}, \\
G_{\mathrm{HC}}\left(\tilde{x}_{1}, \tilde{x}_{2} ; z\right) & =\lim _{\Lambda \nearrow \mathbb{R}^{D}}\left\langle\rho\left(\tilde{x}_{1}\right) \rho\left(\tilde{x}_{2}\right)\right\rangle_{\mathrm{HC}, \Lambda} .
\end{aligned}
$$

Here $\langle\cdot\rangle_{\mathrm{HC}, \Lambda}$ is the expectation in the measure for which $Z_{\mathrm{HC}}(z)$ is the normalizing constant.

Theorem 2.

$$
G_{\mathrm{HC}}(0, x ; z)=-2 \pi \int d^{2} w G_{\mathrm{BP}}\left(0, y ;-\frac{z}{2 \pi}\right),
$$

where $y=(x, w) \in \mathbb{R}^{D+2}$.

Analogous results can be obtained for the $n$-point functions. 


\section{Consequences for Critical Exponents}

One can define a critical exponent $\alpha_{\mathrm{HC}}$ by looking at the singularity of the pressure of the hard-core gas:

$$
\lim _{\Lambda \nearrow \mathbb{R}^{D}} \frac{1}{|\Lambda|} \log Z_{\mathrm{HC}}(z) \sim\left(z-z_{c}\right)^{2-\alpha_{\mathrm{HC}}} .
$$

Likewise, a susceptibility exponent $\gamma_{\mathrm{HC}}$ can be defined from the behavior of $Z_{\mathrm{BP}}$ :

$$
Z_{\mathrm{BP}}\left(-\frac{z}{2 \pi}\right) \sim\left(z-z_{c}\right)^{2-\gamma_{\mathrm{BP}}} .
$$

Keep in mind that $z_{c}$ is negative and $-\frac{z_{c}}{2 \pi}$ is positive-Theorems 1 and relate the critical behavior of branched polymers to the critical behavior of the hard-core gas at negative activity. Theorem 1 equates the two singularities, so

$$
\alpha_{\mathrm{HC}}=\gamma_{\mathrm{BP}} .
$$

Examining the 2-point functions of each model near their critical points, we can define exponents $\eta_{\mathrm{HC}}, \eta_{\mathrm{BP}}$ from the asymptotic forms

$$
\begin{aligned}
G_{\mathrm{HC}}(0, x ; z) & \sim x^{-\left(D-2+\eta_{\mathrm{HC}}\right)} K_{\mathrm{HC}}\left(x / \xi_{\mathrm{HC}}\right), \\
G_{\mathrm{BP}}(0, x ; z) & \sim x^{-\left(d-2+\eta_{\mathrm{BP}}\right)} K_{\mathrm{BP}}\left(x / \xi_{\mathrm{BP}}\right)
\end{aligned}
$$

as $|x| \rightarrow \infty$ with $\hat{x}:=x / \xi$ fixed. Here $d=D+2, \xi_{\mathrm{HC}}, \xi_{\mathrm{BP}}$ are the correlation lengths of the respective systems, and $K_{\mathrm{HC}}, K_{\mathrm{BP}}$ are scaling functions. Theorem 2 implies that $\xi_{\mathrm{HC}}=\xi_{\mathrm{BP}}$, so in particular the exponents $\nu_{\mathrm{HC}}=\nu_{\mathrm{BP}}$, where

$$
\begin{aligned}
& \xi_{\mathrm{HC}}(z) \sim\left(z-z_{c}\right)^{-\nu_{\mathrm{HC}}}, \\
& \xi_{\mathrm{BP}}(z) \sim\left(z-z_{c}\right)^{-\nu_{\mathrm{BP}}}
\end{aligned}
$$

as $z \searrow z_{c}$. Theorem 2 also implies that

$$
\eta_{\mathrm{HC}}=\eta_{\mathrm{BP}}
$$

for $D \geq 1$. Furthermore, if one defines $\theta$ from

$$
Z_{\mathrm{BP}}(z)=\sum_{N=1}^{\infty} c_{N} z^{N}
$$

with

$$
c_{N} \sim z_{c}^{-N} N^{-\theta} .
$$

Then

$$
\theta=3-\gamma_{\mathrm{BP}}
$$


Further results can be obtained for the scaling functions. We can express $K_{\mathrm{BP}}$ in terms of $K_{\mathrm{HC}}$ and its radial derivative:

$$
K_{\mathrm{BP}}(\hat{x})=\frac{1}{4 \pi^{2}}\left[\hat{x} K_{\mathrm{HC}}^{\prime}(\hat{x})-\left(D-2+\eta_{\mathrm{HC}}\right) K_{\mathrm{HC}}(\hat{x})\right] .
$$

In $D=1$ we can compute BI03

$$
K_{\mathrm{HC}}(\hat{x})=-\frac{4}{\hat{x}^{2}} e^{-\hat{x}},
$$

so that in $D+2=3$ dimensions,

$$
K_{\mathrm{BP}}(\hat{x})=\frac{1}{\pi^{2} \hat{x}} e^{-\hat{x}},
$$

which agrees with the prediction of Miller Mil91. Let us tabulate the known or for the critical exponents of the hard-sphere gas in various dimensions.

\begin{tabular}{|c|c|c|c|c|}
\hline$D$ & $\alpha$ & $\nu_{\mathrm{HC}}$ & $\eta_{\mathrm{HC}}$ & $\sigma=1-\alpha$ \\
\hline 0 & 2 & & & -1 \\
\hline 1 & $\frac{3}{2}$ & $\frac{1}{2}$ & -1 & $-\frac{1}{2}$ \\
\hline 2 & $\frac{7}{6}$ & $\frac{5}{12}$ & $-\frac{4}{5}$ & $-\frac{1}{6}$ \\
\hline MFT $D>6$ & $\frac{1}{2}$ & $\frac{1}{4}$ & 0 & $\frac{1}{2}$ \\
\hline
\end{tabular}

In $D=0,1$, the values are rigorous. In $D=2$, the value $\alpha=\frac{7}{6}$ is obtained from Baxter's solution to the hard hexagon model Bax82 Dha83 BL87. The last column gives values for $\sigma$, the Yang-Lee edge exponent. Assuming that the hard-sphere gas at negative activity falls into the Yang-Lee edge universality class, we have $\sigma=1-\alpha$. Actually, from hyperscaling $\left(D \nu_{\mathrm{HC}}=2-\alpha_{\mathrm{HC}}\right)$ and Fisher's relation $\sigma=\frac{D-2+\eta_{\mathrm{HC}}}{D+2-\eta_{\mathrm{HC}}}$, we know that there is only one independent exponent in each dimension. The value $\eta_{\mathrm{HC}}=-\frac{4}{5}$ is independently confirmed from conformal field theory Car85.

The branched polymer critical exponents in $D+2$ dimensions can be equated to the corresponding values for the hard sphere gas in $D$ dimensions; see the first three columns of the following table.

\begin{tabular}{|c|c|c|c|c|}
\hline$d=D+2$ & $\gamma=\alpha$ & $\nu_{\mathrm{BP}}=\nu_{\mathrm{HC}}$ & $\eta_{\mathrm{BP}}=\eta_{\mathrm{HC}}$ & $\theta=3-\gamma$ \\
\hline 2 & 2 & & & 1 \\
\hline 3 & $\frac{3}{2}$ & $\frac{1}{2}$ & -1 & $\frac{3}{2}$ \\
\hline 4 & $\frac{7}{6}$ & $\frac{5}{12}$ & $-\frac{4}{5}$ & $\frac{11}{6}$ \\
\hline MFT $D>8$ & $\frac{1}{2}$ & $\frac{1}{4}$ & 0 & $\frac{5}{2}$ \\
\hline
\end{tabular}


The values in two and three dimensions are now rigorous. Note that in high dimensions $(d>8)$ it has been proven that $\gamma_{\mathrm{BP}}=\frac{1}{2}, \nu_{\mathrm{BP}}=\frac{1}{4}, \eta_{\mathrm{BP}}=0$ (at least for spread-out lattice models) HS90 HS92 HvS03. While our results do not apply to lattice models, they give a strong indication that the corresponding hard-core exponents have the same (mean-field) values for $D>6$.

\section{Forest-Root Formula}

The underlying mechanism behind all these results is an interpolation formula (the Forest-Root formula). Let $f(\mathbf{t})$ depend on $\mathbf{t}=\left(t_{i j}\right),\left(t_{i}\right)$ for $1 \leq i<j \leq N$. Assume that $f \rightarrow 0$ at $\infty$. Let $t_{i j}=\left|w_{i}-w_{j}\right|^{2}, t_{i}=\left|w_{i}\right|^{2}$ with $w_{i} \in \mathbb{C}$. Then

$$
f(\mathbf{0})=\sum_{(F, R)} \int_{\mathbb{C}^{N}} f^{(F, R)}(\mathbf{t})\left(\frac{d^{2} w}{-\pi}\right)^{N} .
$$

The sum is over forests $F$ and roots $R$ (collections of bonds $i j$, and vertices $i$, respectively) such that each tree of $F$ has exactly one root $R$. See Fig 1.

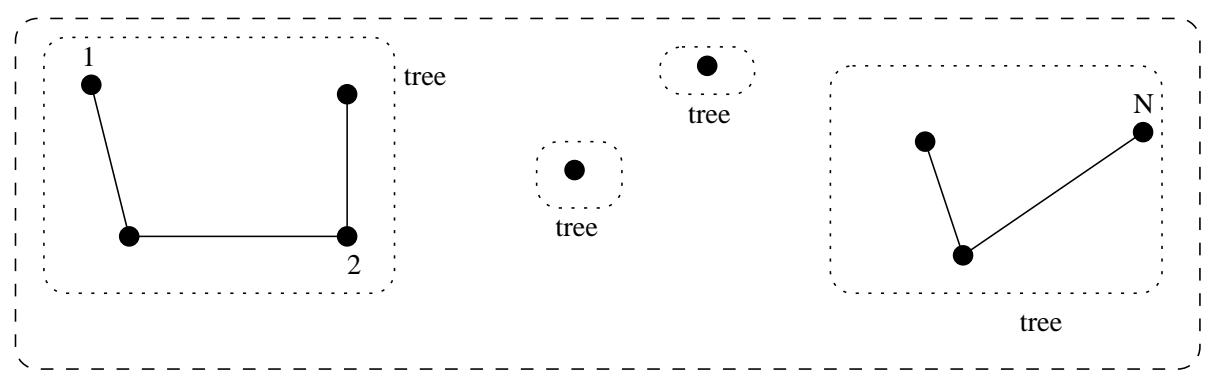

forest

Figure 1: Example of a forest

For a simple example, consider the case $N=1, F=\emptyset, R=\{1\}$. Then the formula reduces to the fundamental theorem of calculus in one variable:

$$
f(0)=\int_{\mathbb{C}} f^{\prime}(t) \frac{d^{2} w}{-\pi}=-\int_{0}^{\infty} f^{\prime}(t) d t .
$$

\section{Supersymmetry}

To understand the Forest-Root formula in general, we need to exploit supersymmetry. Replace in $f(\mathbf{t})$ each variable $t_{i}$ with

$$
\tau_{i}=w_{i} \bar{w}_{i}+\frac{d w_{i} \wedge d \bar{w}_{i}}{2 \pi i},
$$


and $t_{i j}$ with

$$
\tau_{i j}=w_{i j} \bar{w}_{i j}+\frac{d w_{i j} \wedge d \bar{w}_{i j}}{2 \pi i}
$$

(Recall that $\left.w_{i j}=w_{i}-w_{j}\right)$. Then $f(\underline{\tau})$ is defined by its Taylor series. I claim that a "localization" formula holds:

$$
\int_{\mathbb{C}^{N}} f(\underline{\tau})=f(\mathbf{0}) .
$$

This formula becomes the Forest-Root formula when expanded out. The absence of loops comes from the fact that $(d w \wedge d \bar{w})^{G}=0$ if $G$ has a loop. We prove the formula by deforming the problem to the independent case, using ideas from Wit92.

An alternate argument can be given, exploiting the linearity of (34) to reduce to the case where $f$ is an exponential BW88. Let

$$
\hat{f}(\mathbf{a})=\int_{0}^{\infty} d \mathbf{t} f(\mathbf{t}) e^{\mathbf{a} \cdot \mathbf{t}}
$$

be the Laplace transform of $f$. Here $\mathbf{a}=\left(a_{i}\right),\left(a_{i j}\right)$ is dual to $\mathbf{t}$, that is $\mathbf{a} \cdot \mathbf{t}=$ $\Sigma_{i j} a_{i j} t_{i j}+\Sigma_{i} a_{i} t_{i}$. Let us assume that $f$ has exponential decay in each $t_{i}$ variable, so that we can take the $a_{i}$ 's to have positive real part. Cases where $f$ decreases more slowly at infinity can be treated by taking limits. We can write $f$ in terms of its Laplace transform,

$$
f(\mathbf{t})=\int \prod_{i} \frac{d a_{i}}{2 \pi i} \prod_{i j} \frac{d a_{i j}}{2 \pi i} \hat{f}(\mathbf{a}) e^{-\mathbf{a} \cdot \mathbf{t}},
$$

with the integration contours running parallel to the imaginary axis. We see that (34) holds if it is true for functions of the form $e^{-\mathbf{a} \cdot \mathbf{t}}$.

Let us write

$$
\mathbf{a} \cdot \mathbf{t}=\langle w, A \bar{w}\rangle+\left\langle\frac{d w}{\sqrt{2 \pi i}}, A \frac{d \bar{w}}{\sqrt{2 \pi i}}\right\rangle,
$$

where

$$
\begin{aligned}
& A_{i j}=-a_{i j}, \quad i \neq j, \\
& A_{i i}=a_{i}+\sum_{j \neq i} a_{i j} .
\end{aligned}
$$

The matrix $A$ is symmetric and has positive real part. Then by the properties of the differential forms $d w_{i}, d \bar{w}_{i}$,

$$
\begin{aligned}
\int_{\mathbb{C}^{N}} e^{-\mathbf{a} \cdot \mathbf{t}} & =\int_{\mathbb{C}^{N}} e^{-\langle w, A \bar{w}\rangle} \exp \left[-\left\langle\frac{d w}{\sqrt{2 \pi i}}, A \frac{d \bar{w}}{\sqrt{2 \pi i}}\right\rangle\right] \\
& =\int_{\mathbb{C}^{N}} e^{-\langle w, A \bar{w}\rangle} \operatorname{det} A \prod_{i=1}^{N}\left[\frac{-d w_{i} \wedge d \bar{w}_{i}}{2 \pi i}\right] .
\end{aligned}
$$


With $w_{i}=x_{i}+i y_{i}$, we have that $d w_{i} \wedge d \bar{w}_{i}=-2 i d x_{i} \wedge d y_{i}$, so this is

$$
\pi^{-N} \operatorname{det} A \int e^{-\langle w, A \bar{w}\rangle} d^{N} x d^{N} y=1,
$$

and we obtain (34) for $f(\mathbf{t})=e^{-\mathbf{a} \cdot \mathbf{t}}$.

\section{Decoupling in Two Extra Dimensions}

Use the Forest-Root formula to decouple the spheres in the hard-core gas by moving them apart in two extra dimensions. At fixed $\mathrm{N}$ we have an integral over $\mathbf{x} \in \Lambda^{N} \subset \mathbb{R}^{D N}$ of

$$
f(\mathbf{0})=\prod_{1 \leq i<j \leq N} U\left(\left|x_{i j}\right|^{2}\right),
$$

where $t_{i}=\left|w_{i}\right|^{2}=0$. Extend this to $w \neq 0$ by writing

$$
f(\mathbf{t})=\prod_{1 \leq i<j \leq N} U\left(\left|x_{i j}\right|^{2}+t_{i j}\right) \times(\text { large } t \text { cutoff }) .
$$

Apply the Forest-Root formula,

$$
f(\mathbf{0})=\sum_{(F, R)} \int_{\mathbb{C}^{N}} f^{(F, R)}(\mathbf{t})\left(\frac{d^{2} w}{-\pi}\right)^{N} .
$$

Then each $\frac{d}{d t_{i j}}$, when applied to $U_{i j}$, becomes $\frac{1}{2}$ surface measure for the combined integrals over $y_{i j}=\left(x_{i j}, w_{i j}\right)$. This means that spheres are stuck together according to the forest $F$. Furthermore, the trees of the forest decouple in the limit as the large $t$ cutoff is removed. This is because they are spread apart in the $w$ directions, so that they rarely interact. As a result, $Z_{\mathrm{HC}}$ can be written in the form of an exponential of tree graphs. Thus $\log Z_{\mathrm{HC}}$ is evaluated as a sum/integral over branched polymers, and Theorem $\square$ is proven. See BI01 for details.

\section{Relation with the Yang-Lee Edge}

Repulsive gases near criticality at negative activity are described by an $i \varphi^{3}$ field theory. More precisely, they should fall into the same universality class - see Car82 Dha83 LF95 PF99. To see this, replace the hard-core potential with a repulsive, smooth, rapidly decaying two-body potential

$$
u\left(x_{i j}\right)=v\left(\left|x_{i j}\right|^{2}\right) \text { with } \hat{u}(k)>0 .
$$

By the sine-Gordon transformation, the repulsive gas

$$
Z_{v}(z)=\sum_{N=0}^{\infty} \frac{z^{N}}{N !} \int_{\Lambda^{N}} d x_{1} \cdots d x_{N} \exp \left[-\beta \sum_{1 \leq i<j \leq N} u\left(x_{i j}\right)\right]
$$


can be written as a $-\tilde{z} e^{i \varphi}$ field theory. With $\tilde{z}=z e^{\beta v(0) / 2}$, we have

$$
Z_{v}(z)=\int \exp \left[\int_{\Lambda} d x \tilde{z} e^{i \varphi(x)}\right] \frac{e^{-\frac{1}{2}\left\langle\varphi,(\beta u)^{-1} \varphi\right\rangle}[d \varphi]}{\mathcal{N}} .
$$

If $\tilde{z}$ is negative enough, this action looks critical. In fact at the critical value of $\tilde{z}$, the lowest order term in the action is $i \varphi^{3}$. A similar situation occurs for Ising or $\varphi^{4}$ model in a sufficiently large imaginary field [Fis78.

\section{Dimensional Reduction: Soft Branched Polymers}

The arguments leading to Theorems 1 and work for any $U(t)$ for which $U-1$ and $U^{\prime}$ have sufficient decay. Take

$$
U(t)=\exp (-\beta v(t))
$$

to get the identity

$$
\lim _{\Lambda \nearrow \mathbb{R}^{D}} \frac{1}{|\Lambda|} \log Z_{v}(z)=-2 \pi Z_{\mathrm{BP}, v}\left(-\frac{z}{2 \pi}\right),
$$

where $Z_{\mathrm{BP}, v}$ is given by the same formula 4 as before but with this $U$. In this model of "soft" branched polymers, the repulsion between monomers is given by a factor $\exp \left(-\beta v\left(\left|x_{i j}\right|^{2}\right)\right)$, and the attraction linking neighboring monomers in the tree graph is given by a factor $\left.-2 \beta v^{\prime}\left(\left|x_{i j}\right|^{2}\right)\right)$. Together with the sine-Gordon representation for $Z_{\mathrm{BP}, v}$, we obtain a direct representation for branched polymers in $D+2$ dimensions as a $-\tilde{z} e^{i \varphi}$ field theory in $D$ dimensions. As this is presumably in the Yang-Lee class, we have confirmed the prediction of Parisi and Sourlas PS81.

\section{Crossover to Mean-Field Branched Polymers}

We wish to understand what happens as the repulsion between monomers is turned off. In the limit, we obtain a mean-field or noninteracting model (on the lattice such models were analyzed in Frö86 BCvS). Let $v=v(0)$ be small and $z$ be large so that a redefined $\tilde{z}=z v e^{v / 2}$ is fixed. In these variables (50) becomes

$$
\log \int_{-\infty}^{\infty} \exp \left[-\frac{1}{v}\left(\tilde{z} e^{i \varphi}+\frac{1}{2} \varphi^{2}\right)\right] \frac{d \varphi}{\sqrt{2 \pi v}}=-2 \pi Z_{\mathrm{BP}, v}\left(\frac{z}{2 \pi}\right) .
$$

I have reversed the sign of $z$, so the branched polymer singularity is now at positive $z$.

It should be clear that the $v \rightarrow 0$ limit simplifies both sides of this identity. On the 0 -dimensional Yukawa gas side, $v \rightarrow 0$ means the integral reduces to finding the critical point of $\tilde{z} e^{i \varphi}+\frac{1}{2} \varphi^{2}$. On the two-dimensional branched polymer side, $v \rightarrow 0$ eliminates the self-avoidance. Every tree has one fewer edges than vertices. 
Assuming $v^{\prime}$ remains proportional to $v$, as $v \rightarrow 0$ and $z v \rightarrow \tilde{z}$, this means that $\frac{d}{d z} Z_{\mathrm{BP}, v}$ goes to a finite limit, which is a generating function of non-self-avoiding (or mean-field) branched polymers containing the origin.

We can observe the crossover by considering $Z_{\mathrm{BP}, v}$ as a function of $\tilde{z}$ and $v$ near $v=0$ and $\tilde{z}=\tilde{z}_{c}$. With $S(\varphi)=\frac{1}{2} \varphi^{2}+\tilde{z} e^{i \varphi}$, we have

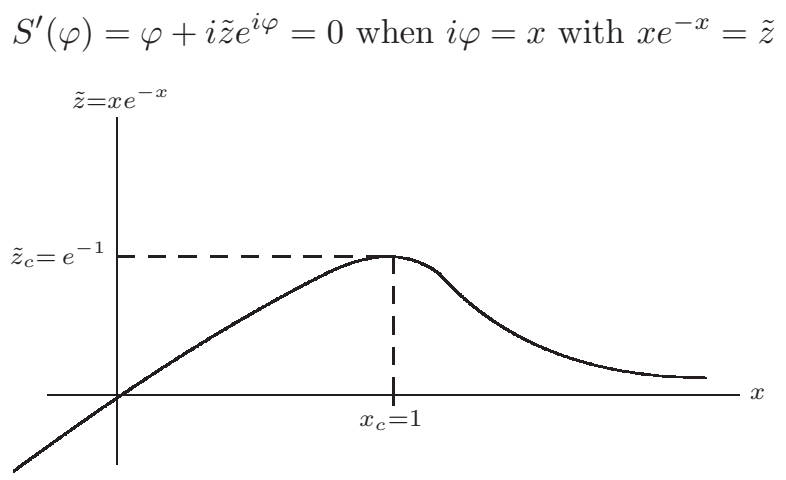

The solution is $x=x(\tilde{z})=T(\tilde{z})$ as in the graph. We also solve

$$
S^{\prime \prime}(\varphi)=1-\tilde{z} e^{i \varphi}=0 \text { when } \tilde{z}=e^{-i \varphi}=e^{-x}=\frac{\tilde{z}}{x},
$$

so $x_{c}=1, \tilde{z}_{c}=e^{-1}$ has $S^{\prime}=S^{\prime \prime}=0$.

Let us expand $S(\varphi)$ about $\varphi=-i$ with $\tilde{z}$ close to $\tilde{z}_{c}=e^{-1}$. With $\tilde{\varphi}:=\varphi+i$ we obtain

$$
\begin{aligned}
S(\tilde{\varphi}-i) & =\frac{1}{2} \tilde{\varphi}^{2}-i \tilde{\varphi}-\frac{1}{2}+e \tilde{z} e^{i \tilde{\varphi}} \\
& =\frac{1}{2} \tilde{\varphi}^{2}-i \tilde{\varphi}-\frac{1}{2}-e\left(z_{c}-z\right) e^{i \tilde{\varphi}}+e^{i \tilde{\varphi}} .
\end{aligned}
$$

Let $t=\frac{\tilde{z}_{c}-\tilde{z}}{\tilde{z}_{c}}$. This becomes

$$
\left(e^{i \tilde{\varphi}}-1-i \tilde{\varphi}+\frac{1}{2} \tilde{\varphi}^{2}\right)-t\left(e^{i \tilde{\varphi}}-1\right)-t+\frac{1}{2}=-\frac{i \tilde{\varphi}^{3}}{6}-i t \tilde{\varphi}+O\left(\tilde{\varphi}^{4}\right)+t O\left(\tilde{\varphi}^{2}\right)-t+\frac{1}{2},
$$

and so from 517

$$
-2 \pi Z_{\mathrm{BP}, v}\left(\frac{z}{2 \pi}\right)=\log \int \exp \left[\frac{1}{v}\left(\frac{i \tilde{\varphi}^{3}}{6}+i t \tilde{\varphi}+O\left(\tilde{\varphi}^{4}\right)+t O\left(\tilde{\varphi}^{2}\right)+t-\frac{1}{2}\right)\right] \frac{d \tilde{\varphi}}{\sqrt{2 \pi v}} .
$$

Let us apply $\frac{d}{d z}$ to get the sum of branched polymers containing the origin. With $G_{\mathrm{BP}, v}(y)=\frac{d}{d y} Z_{\mathrm{BP}, v}(y)$, we have

$$
G_{\mathrm{BP}, v}\left(\frac{z}{2 \pi}\right)=-\frac{d}{d z} \log \int \exp \left[\frac{1}{v}\left(\frac{i \tilde{\varphi}^{3}}{6}+i t \tilde{\varphi}+O\left(\tilde{\varphi}^{4}\right)+t O\left(\tilde{\varphi}^{2}\right)+t\right)\right] d \tilde{\varphi} .
$$

The expression in the exponent is useful for $\tilde{\varphi}$ small. Consider the crossover regime, $|t| \leq O\left(v^{2 / 3}\right)$. I wish to show that the contribution to the integral from 
$|\tilde{\varphi}|>v^{1 / 3-\epsilon}$ is smaller than any power of $v$. Make a kink in the contour of integration for $\tilde{\varphi}$, so that it lies at angles $\frac{\pi}{6}$ and $\frac{5 \pi}{6}$. This turns $i \tilde{\varphi}^{3}$ into $-|\tilde{\varphi}|^{3}$. From [55], one can check that on this contour,

$$
\operatorname{Re} S(\tilde{\varphi}) \geq \text { const } \min \left\{|\tilde{\varphi}|^{3},|\tilde{\varphi}|^{2}\right\}-t O(\tilde{\varphi})-t+\frac{1}{2},
$$

so that $\tilde{\varphi}$ is effectively of order $v^{1 / 3}$. This means that $\frac{1}{v} O\left(\tilde{\varphi}^{4}\right)$ and $\frac{t}{v} O\left(\tilde{\varphi}^{2}\right)$ are $O\left(v^{1 / 3}\right)$ and that these terms do not contribute to $Z_{\mathrm{BP}, v}$ to leading order in $v^{1 / 3}$. Likewise when computing $\frac{d}{d z}=v e^{v / 2} \frac{d}{d \tilde{z}}=-e v e^{v / 2} \frac{d}{d t}$ in [57), the term $O\left(\tilde{\varphi}^{2}\right)=$ $O\left(v^{2 / 3}\right)$ is subleading order. The term $-\frac{d}{d z} \frac{t}{v}=e^{1+v / 2}$ is nonsingular. Hence the singular behavior of $G_{\mathrm{BP}, v}$ is given to leading order by

$$
G_{\mathrm{BP}, v}^{(\mathrm{sing})}\left(\frac{z}{2 \pi}\right)=-\frac{d}{d z} \log \int \exp \left[\frac{1}{v}\left(\frac{i \tilde{\varphi}^{3}}{6}+i t \tilde{\varphi}\right)\right] d \tilde{\varphi} .
$$

We have arrived at a point very similar to the one Cardy reached in his analysis of the crossover from area-weighted self-avoiding loops to self-avoiding loops Car01. He related that problem to branched polymers and, as we have done here, used dimensional reduction to compute the crossover scaling function as an Airy integral. If we rescale $\varphi \rightarrow \varphi v^{-1 / 3}$ in 59, we obtain as in Car01 that

$$
G_{\mathrm{BP}, v}^{(\text {sing })}=v^{\frac{1}{3}} F\left(t v^{-\frac{2}{3}}\right),
$$

where

$$
F(s)=b_{0} \frac{d}{d s} \ln \operatorname{Ai}\left(b_{1} s\right) .
$$

Here $b_{0}$ and $b_{1}$ are constants, and $\operatorname{Ai}(t)=\frac{1}{2 \pi} \int_{-\infty}^{\infty} e^{i t \varphi+i \varphi^{3} / 3} d \varphi$ is the Airy function.

The expression (60) interpolates between two different critical behaviors for branched polymers. We have already seen that $\gamma_{\mathrm{BP}}=1$ in two dimensions; this corresponds to the pole in 600 that occurs when $v$ is fixed and nonzero, and $t$ decreases through 0 to the first zero of $\operatorname{Ai}\left(b_{1} t v^{-\frac{2}{3}}\right)$. This is the limit of convergence of $Z_{\mathrm{BP}}$; past this point [51] no longer makes sense.

If, instead, we send $v$ to zero and then let $t \searrow 0$, this means $\tilde{\varphi}$ is replaced by its critical value $\tilde{\varphi}_{c}$ where $S^{\prime}\left(\tilde{\varphi}_{c}-i\right)=0$, c.f. [52). Then,

$$
\begin{aligned}
\lim _{v \rightarrow 0} G_{\mathrm{BP}, v}\left(\frac{z}{2 \pi}\right) & =\lim _{v \rightarrow 0}-v e^{v / 2} \frac{d}{d \tilde{z}} \log \int \exp \left[-\frac{1}{v}\left(\tilde{z} e^{i \tilde{\varphi}}+\frac{1}{2} \tilde{\varphi}^{2}\right)\right] d \tilde{\varphi} \\
& =e^{i \tilde{\varphi}_{c}} .
\end{aligned}
$$

It is clear from the graph above that $i \tilde{\varphi}_{c} \sim-t^{1 / 2}$. Thus the leading singularity of [62] is $t^{1-\gamma}=t^{1 / 2}$, and $\gamma=\frac{1}{2}$. This agrees with the value determined for the mean field models in Frö86 BCvS. This also agrees with the conjectured value of the entropic exponent of self-avoiding walks. The square-root behavior can be seen in 601so, as $F(s) \sim s^{1 / 2}$ as $s \nearrow \infty$. 
The identity of the crossover scaling functions and critical exponents for selfavoiding loops and branched polymers suggests a close connection between the two systems. It is natural to think of loops arising as the frontier of branched polymers. In this picture, the self-avoidance interaction of the branched polymer gives rise to an effective area weighting for the loop, and the mean-field limit of branched polymers corresponds to loops without area weighting. This fits in with recent results LSW01 on the Hausdorff dimension of the frontier of Brownian motion (suggesting a link with self-avoiding walks).

\section{Acknowledgement}

The author wishes to acknowledge conversations with David Brydges, John Cardy, Paul Fendley, and Yonathan Shapir, who helped shape my understanding of these problems.

\section{References}

[Bax82] R. J. Baxter, Exactly solved models in statistical mechanics, Academic Press Inc. [Harcourt Brace Jovanovich Publishers], London, 1982.

[BCvS] C. Borgs, J. Chayes, R. van der Hofstad, and G. Slade, Mean-field lattice trees, Ann. Comb. 3, 205-221 (1999), arXiv:math.PR/9904184

[BD98] E. Brézin and C. De Dominicus, New phenomena in the random field Ising model, Europhys. Lett. 44, 13-19 (1998), arXiv:cond-mat/9804266

[BI01] D.C. Brydges and J.Z. Imbrie, Branched polymers and dimensional reduction, preprint, arXiv:math-ph/0107005

[BI03] D.C. Brydges and J.Z. Imbrie, Dimensional reduction formulas for branched polymer correlation functions, J. Statist. Phys. 110, 503-518 (2003), arXiv:math-ph/0203055

[BL87] A. Baram and M. Luban, Universality of the cluster integrals of repulsive systems, Phys. Rev. A 36, 760-765 (1987).

[BW88] D. C. Brydges and J. Wright, Mayer expansions and the HamiltonJacobi equation. II. Fermions, dimensional reduction formulas, $J$. Statist. Phys. 51, 435-456 (1988). Erratum: J. Statist. Phys. 97, 1027 (1999).

[Car82] J. L. Cardy, Directed lattice animals and the Lee-Yang edge singularity, J. Phys. A 15, L593-L595 (1982).

[Car85] J. L. Cardy, Conformal invariance and the Yang-Lee edge singularity in two dimensions, Phys. Rev. Lett. 54, 1354-1356 (1985). 
[Car01] J.L. Cardy, Exact scaling functions for self-avoiding loops and branched polymers, J. Phys. A 34, L665-L672 (2001), arXiv:cond-mat/0107223

[CGHJK] R. M. Corless, G. H. Gonnet, D. E. G. Hare, D. J. Jeffrey, and D. E. Knuth, On the Lambert $W$ function, Adv. Comput. Math. 5, 329-359 (1996).

[Dha83] D. Dhar, Exact solution of a directed-site animals-enumeration problem, Phys. Rev. Lett. 51, 853-856 (1983).

[Fis78] M. E. Fisher, Yang-Lee edge singularity and $\varphi^{3}$ field theory, Phys. Rev. Lett. 40, 1610-1613 (1978).

[Fel02] D. E. Feldman, Critical exponents of the random-field $O(N)$ model, Phys. Rev. Lett. 88, 177202 (2002), arXiv:cond-mat/0010012

[Frö86] J. Fröhlich, Mathematical aspects of the physics of disordered systems. In Phénomènes critiques, systèmes aléatoires, théories de jauge, Part II (Les Houches, 1984), Amsterdam: North-Holland, 1986, pp. 725-893.

[HS90] T. Hara and G. Slade, On the upper critical dimension of lattice trees and lattice animals, J. Statist. Phys. 59, 1469-1510 (1990).

[HS92] T. Hara and G. Slade, The number and size of branched polymers in high dimensions, J. Statist. Phys. 67, 1009-1038 (1992).

[HvS03] T. Hara, R. van der Hofstad, and G. Slade, Critical two-point functions and the lace expansion for spread-out high-dimensional percolation and related models, Ann. Probab. 31, 349-408 (2003), arXiv:math-ph/0011046

[Imb84] J. Z. Imbrie, Lower critical dimension of the random-field Ising model, Phys. Rev. Lett. 53, 1747-1750 (1984).

[Imb85] J. Z. Imbrie, The ground state of the three-dimensional random-field Ising model, Commun. Math. Phys. 98, 145-176 (1985).

[LF95] S. Lai and M. E. Fisher, The universal repulsive-core singularity and Yang-Lee edge criticality, J. Chem. Phys. 103, 8144-8155 (1995).

[LSW01] G. F. Lawler, O. Schramm, and W. Werner, The Dimension of the Planar Brownian Frontier is 4/3, Math. Res. Lett. 8, 401-411 (2001), arXiv:math.PR/0010165

[Mil91] J. D. Miller, Exact pair correlation function of a randomly branched polymer, Europhys. Lett. 16, 623-628 (1991). 
[PF99] Y. Park and M. E. Fisher, Identity of the universal repulsive-core singularity with Yang-Lee edge criticality, Phys. Rev. E 60, 6323-6328 (1999), arXiv:cond-mat/9907429

[PS79] G. Parisi and N. Sourlas, Random magnetic fields, supersymmetry and negative dimensions, Phys. Rev. Lett. 43, 744-745 (1979).

[PS81] G. Parisi and N. Sourlas, Critical behavior of branched polymers and the Lee-Yang edge singularity, Phys. Rev. Lett. 46, 871-874 (1981).

[PS02] G. Parisi and N. Sourlas, Scale invariance in disordered systems: the example of the random-field Ising model, Phys. Rev. Lett. 89, 257204 (2002), arXiv:cond-mat/0207415

[Sha83] Y. Shapir, Supersymmetric dimer Hamiltonian for lattice branched polymers, Phys. Rev. A 28, 1893-1895 (1983).

[Sha85] Y. Shapir, Supersymmetric statistical models on the lattice, Physica D 15, 129-137 (1985).

[Wit92] Edward Witten, Two-dimensional gauge theories revisited, J. Geom. Phys. 9, 303-368 (1992), arXiv:hep-th/9204083 